\title{
Supersymmetry and the Systematics of T-duality Rotations in Type-II Superstring Theories
}

\author{
S. F. Hassan * $\dagger$ \\ Helsinki Institute of Physics, P.O. Box 64, FIN-00014, University of Helsinki, Finland
}

We describe a systematic method of studying the action of the T-duality group $O(d, d)$ on space-time fermions and R-R field strengths and potentials in type-II string theories, based on space-time supersymmetry. The formalism is then used to show that the couplings of non-Abelian D-brane charges to R-R potentials can be described by an appropriate Clifford multiplication.

Report No: HIP-2001-07/TH, hep-th/010314

\section{Introduction:}

Let us denote the massless NS-NS background fields by $G_{M N}, B_{M N}$ and $\phi$, and the R-R $n$-form field strengths by $F_{M_{1} \cdots M_{n}}^{(n)}$, where $n$ is even in type-IIA and odd in type-IIB theory. It is customary to define two sets of R-R potentials, which we denote by $C_{M_{1} \cdots M_{n}}^{(n)}$ and $C_{M_{1} \cdots M_{n}}^{(n)}$. Denoting by $\mathbf{F}$ and $\mathbf{C}$ the sums of $n$-forms $\sum_{n=1}^{n=9} F^{(n)}$ and $\sum_{n=0}^{n=8} C^{(n)}$ (similarly for $\mathbf{C}^{\prime}$ ), the relations among them can be written as

$\mathbf{F}=d \mathbf{C}-H \wedge \mathbf{C}=\mathrm{e}^{B} \wedge d \mathbf{C}^{\prime}$

Here, $H=d B$ and the two sets of potentials are related by $\mathbf{C}^{\prime}=\mathbf{C} \wedge \mathrm{e}^{-B}$. Also we denote the two gravitinos, the two dilatinos and two supersymmetry transformation parameters by $\Psi_{ \pm M}$, $\lambda_{ \pm}$and $\epsilon_{ \pm}$, respectively. In type-IIA theory the fermions labelled by \pm have opposite chiralities, while in type-IIB they have the same chirality.

Let $X^{M},(M=0, \cdots 9)$ denote the space-time coordinates and consider background field configurations which are independent of the $d$ coordinates $X^{i},(i=1, \cdots d)$, but may vary with the remaining $10-d$ coordinates $X^{\mu}, \mu=0, d+1, \cdots 9$. The T-duality group $O(d, d)$ acts on these backgrounds such that the equations of motion, when restricted to $X^{i}$-independent fields, remain invariant. The transformation of NS-NS fields un-

\footnotetext{
*fawad.hassan@helsinki.fi

†Contribution to the proceedings of the D. V. Volkov memorial conference on "Supersymmetry and Quantum Field Theory", Kharkov, July 25-29, 2000 (to appear in the Nucl. Phys. B Conference Supplements).
}

der this group is well known and can be obtained in a variety of ways. Here we describe a systematic procedure, developed in [1.21, for obtaining the transformations of R-R field strengths and potentials. As a by-product, one also obtains the transformations of the gravitinos, dilatinos and supersymmetry parameters. In the last section, we describe an application of the results to the coupling of non-Abelian D-brane charges to R-R backgrounds [3].

The transformation of R-R potentials $C^{(n)}$ under a single T-duality was first obtained in [4] in the effective low-energy theory. More recently, the case of single T-duality in the presence of supersymmetry has also been considered in [5-7]. In [8,9] it was observed that the potentials $C^{\prime(n)}$ transform in the spinor representations of the $\mathrm{T}$ duality group, verified directly in [10]. Also, see [11. However, this cannot be used to obtain the transformation of $C^{(n)}$ and $F^{(n)}$ under the nontrivial elements of the full T-duality group.

\subsection{Supersymmetry Transformations}

We obtain the transformations of massless R-R and R-NS fields under non-trivial $O(d, d)$ transformations, by demanding compatibility between T-duality and space-time supersymmetry. In type-II theories, this leads to the invariance of the equations of motion.

Not all terms in the supersymmetry transformations are needed for the analysis. To see the basic structure, let us first consider type-II superstring theories in flat space. The two space-time supersymmetry transformations $\delta_{ \pm}$act indepen- 
dently on the left $(+)$ and right $(-)$ moving sectors of the worldsheet by interchanging Ramond states $\left|R_{ \pm}\right\rangle$with Neveu-Schwarz states $\left|N S_{ \pm}\right\rangle$. For example, schematically, we can write

$$
\begin{aligned}
\delta_{-}\left|N S_{+}, R_{-}\right\rangle & =\left|N S_{+}, N S_{-}\right\rangle \epsilon_{-}, \\
\delta_{+}\left|R_{+}, N S_{-}\right\rangle & =\left|N S_{+}, N S_{-}\right\rangle \epsilon_{+}, \\
\delta_{-}\left|R_{+}, N S_{-}\right\rangle & =\left|R_{+}, R_{-}\right\rangle \epsilon_{-} .
\end{aligned}
$$

If we know the action of the T-duality group on the NS-NS sector, then, demanding compatibility with supersymmetry, the first two equations determine the T-duality transformations of the RNS sector and those of $\epsilon_{ \pm}$. One can then use the third equation to determine the T-duality transformation of the R-R sector.

In the presence of background fields, the supersymmetry transformations are more complicated. However, terms with the lowest powers of fermions still retain the flat-space structure. For example, for the gravitinos $\Psi_{ \pm M}$, corresponding to the above three equations we have [12, 13, 1]

$$
\begin{aligned}
\delta_{-} \Psi_{-M} & =\left(\partial_{M}+\frac{1}{4} W_{M a b}^{-} \Gamma^{a b}\right) \epsilon_{-}+\cdots, \\
\delta_{+} \Psi_{+M} & =\left(\partial_{M}+\frac{1}{4} W_{M a b}^{+} \Gamma^{a b}\right) \epsilon_{+}+\cdots, \\
\delta_{-} \Psi_{+M} & =\frac{1}{2(8)} e^{\phi} F \Gamma_{M} \epsilon_{-}+\cdots .
\end{aligned}
$$

Here, $W_{M a b}^{ \pm}=w_{M a b}^{ \pm} \mp \frac{1}{2} H_{M a b}$ are the torsionful spin-connections and $a, b$ are the 10-dimensional Lorentz frame indices. F is a bispinor constructed out the R-R field strengths as

$$
\mathbf{F}=\sum_{n} \frac{(-1)^{n}}{n !} F_{M_{1} \cdots M_{n}}^{(n)} \Gamma^{M_{1} \cdots M_{n}} .
$$

The dots "..." in equations (2)-(4) represent terms containing cubic powers of the spinors. We do not write these out explicitly but emphasize that their contribution will be accounted for in the final results. The strategy now is to first work out the transformation of $W_{M a b}^{ \pm}$under nontrivial elements of the duality group. Then from equations (2) and (3) we obtain the corresponding transformation of $\Psi_{ \pm M}$ and $\epsilon_{ \pm}$. In turn, these can be used in (बi) to find the transformation of $F$. Let us first review the transformation of the metric under the duality group.

\subsection{T-duality of the Metric}

Not all elements of the T-duality group $O(d, d)$ transform the background fields non-trivially. In fact, $G L(d, R)$ transformations of the coordinates $X^{i}$, as well as constant shifts in $B_{i j}$ are subgroups of the T-duality group. These are the trivial elements. The rest act non-trivially and are symmetries of the equations of motion only when the fields do not depend on the $d$ coordinates $X^{i}$. Let us parameterize the $O(d) \times O(d)$ subgroup of $O(d, d)$ by matrices $\mathcal{S} \in O(d)$ and $\mathcal{R} \in O(d)$. All non-trivial elements fall in this subgroup and are parameterized by $\mathcal{S}$ and $\mathcal{R}$ with $\mathcal{S} \neq \mathcal{R}$. The case $\mathcal{S}=\mathcal{R}(=\mathcal{O}$, say $)$ is equivalent to a coordinate transformation $X^{i} \rightarrow \mathcal{O}_{j}^{i} X^{j}$ and belongs to the $O(d)$ subgroup of $G L(d, R)$, already classified as trivial. We will be interested in the transformation of fields under the $O(d) \times O(d)$ subgroup parameterized by the $d$-dimensional matrices $\mathcal{S}, \mathcal{R}$. In order to write the transformation of the 10dimensional fields in a compact way, it is convenient to enlarge these to 10 -dimensional matrices $S$ and $R$ simply by adding the identity matrix for the extra dimensions. By definition, these satisfy $S_{K}^{M} \hat{\eta}^{K L} S_{L}^{N}=\hat{\eta}^{M N}$, similarly for $R$. Here $\hat{\eta}$ is the flat metric with respect to which the orthogonal groups are defined.

The transformation of the metric $G_{M N}$ under the $O(d) \times O(d)$ subgroup can be written in two equivalent ways [14],

$\widetilde{G}^{-1}=Q_{-} G^{-1} Q_{-}^{T}=Q_{+} G^{-1} Q_{+}^{T}$,

where the matrices $Q_{ \pm N}^{M}$ are given by

$$
\begin{aligned}
Q_{-} & =\frac{1}{2}\left[(S+R)+(S-R) \eta^{-1}(G+B)\right], \\
Q_{+} & =\frac{1}{2}\left[(S+R)-(S-R) \eta^{-1}(G-B)\right] .
\end{aligned}
$$

From the structure of $S$ and $R$ one can see that

$\left(Q_{ \pm}\right)_{j}^{\mu}=0, \quad\left(Q_{ \pm}\right)_{\nu}^{\mu}=\delta_{\nu}^{\mu}$,

with similar equations for $Q_{ \pm}^{-1}$. We do not write down the transformations of other NS-NS fields.

\subsection{The Local Lorentz Twist}

To couple fermions to gravity one introduces vielbeins $e_{a}^{M}$ through $G^{M N}=e_{a}^{M} \eta^{a b} e_{b}^{N}$. The observation that the T-duality transformation of 
the metric can be written in two equivalent ways means that there are two possible vielbeins in the dual theory,

$\widetilde{e}_{(-) a}^{M}=Q_{-N}^{M} e_{a}^{N}, \quad \widetilde{e}_{(+) a}^{M}=Q_{+N}^{M} e_{a}^{N}$.

They are related by a Lorentz transformation $\Lambda_{b}^{a}$,

$\widetilde{e}_{(+) b}^{M}=\widetilde{e}_{(-) a}^{M} \Lambda_{b}^{a}, \quad \Lambda_{b}^{a}=e_{M}^{a}\left(Q_{-}^{-1} Q_{+}\right)_{N}^{M} e_{b}^{N}$.

In string theory, the vielbeins $e_{M}^{a}$ and the associated local Lorentz frame can originate in either the right-, or the left- moving sector of the worldsheet theory. Depending on this origin, the vielbeins transform to either $\widetilde{e}_{-}$or $\widetilde{e}_{+}$. Alternatively, as implied by (11), we can choose a single set of vielbeins, say, $\widetilde{e}_{(-)}$, for both the right and left moving sectors, but assume that the associated local Lorentz frames are now twisted with respect to each other by an amount $\Lambda_{b}^{a}$. Below, we show that this local Lorentz twist, induced by the action of the T-duality group, can be undone by absorbing it into the Ramond sector. This, in turn, dictates the transformation of the space-time spinors and R-R fields.

\section{T-duality and Supersymmetry}

Now we have the ingredients needed to analyse the effect of T-duality on the supersymmetry variations (2)-(4). First we need the transformation of the spin-connections $W_{M}^{ \pm}$. In the dual theory, we denote these by $\widetilde{W}_{(-) M}^{ \pm}$. The subscript (-) indicates that they are defined with respect to $\widetilde{e}_{(-)}$. One can show that [1,2]

$\widetilde{W}_{(-) M a b}^{-}=W_{N a b}^{-}\left(Q_{+}^{-1}\right)^{N}$,

$\widetilde{W}_{(+) M a b}^{+}=W_{N a b}^{+}\left(Q_{-}^{-1}\right)_{M}^{N}$.

$\widetilde{W}_{(-) M}^{+}$can now be easily obtained from $\widetilde{W}_{(+) M}^{+}$ by noting that $\widetilde{e}_{(+)}$and $\widetilde{e}_{(-)}$are related by a Lorentz transformation $\Lambda$ (11). Let $\Omega$ denote the spinor representation of $\Lambda$ defined by

$\Omega^{-1} \Gamma^{a} \Omega=\Lambda_{b}^{a} \Gamma^{b}$.

Then, using (96)-(14), one sees that the duality transformations of the spinorial covariant derivatives, $D_{M}^{ \pm}=\partial_{M}+\frac{1}{4} W_{M a b}^{ \pm} \Gamma^{a b}$ are given by

$\widetilde{D}_{(-) M}^{-}=\left(Q_{+}^{-1}\right)^{N}{ }_{M} D_{N}^{-}$,
$\widetilde{D}_{(-) M}^{+}=\Omega \widetilde{D}_{(+) M}^{+} \Omega^{-1}=\left(Q_{-}^{-1}\right)_{M}^{N} \Omega D_{N}^{+} \Omega^{-1} .(16$

\subsection{Transformation of Spinors}

Using the transformation of the spinorial covariant derivatives above, in the supersymmetry variations (2) and (3), one obtains the T-duality action on the supersymmetry parameters $\epsilon_{ \pm}$as

$\widetilde{\epsilon}_{-}=\epsilon_{-}, \quad \widetilde{\epsilon}_{+}=\Omega \epsilon_{+}$.

As for $\Psi_{ \pm M}$, it seems that their transformation is determined only up to terms cubic in the spinors. There are two sources of 3 -spinor corrections: i) equations (2) and (3) contain 3-spinor terms that we have not taken into account, ii) the Tduality transformations $\Psi_{ \pm M}$ and $\delta_{ \pm} \Psi_{ \pm M}$ differ by 3 -spinor terms since $\delta_{ \pm} Q_{\mp}$ and $\delta_{ \pm} \Omega$ are both quadratic in the spinors. It turns out that contributions of type $i$ and $i i$ cancel each other and the T-duality action on the gravitinos is given by

$\widetilde{\Psi}_{-M}=\Psi_{-N}\left(Q_{+}^{-1}\right)^{N}{ }_{M}$,

$\widetilde{\Psi}_{+M}=\Omega \Psi_{+N}\left(Q_{-}^{-1}\right)^{N}$.

The absence of 3-spinor corrections can be confirmed by verifying that they are not allowed by the supersymmetry variations of the NS-NS fields. This means that T-duality does not mix terms with different space-time fermion numbers.

A similar analysis of the supersymmetry variations of the dilatinos gives

$\tilde{\lambda}_{-}=\lambda_{-}-\frac{1}{2} \Gamma_{i}\left[Q_{-}^{-1}(S-R) \hat{\eta}^{-1}\right]^{i j} \Psi_{-j}$,
$\widetilde{\lambda}_{+}=\Omega\left(\lambda_{+}+\frac{1}{2} \Gamma_{i}\left[Q_{+}^{-1}(S-R) \hat{\eta}^{-1}\right]^{i j} \Psi_{+j}\right)$.

\subsection{The R-R Field Strength Bispinor}

In the $O(d, d)$ transformed theory, the supersymmetry variation (1) becomes

$\delta_{-} \widetilde{\Psi}_{+M}=\frac{1}{2(8)} e^{\widetilde{\phi}} \widetilde{F} \widetilde{\Gamma}_{(-) M} \widetilde{\epsilon}_{-}+\cdots$.

Then, using (17) and (19) along with the dilaton transformation, $e^{\phi-\widetilde{\phi}}=\sqrt{\operatorname{det} Q_{-}}$, one obtains

$\widetilde{F}=\sqrt{\operatorname{det} Q_{-}} \Omega \not F$,

where now,

$\widetilde{\mathrm{F}}=\sum_{n} \frac{(-1)^{n}}{n !} \widetilde{F}_{N_{1} \cdots N_{n}}^{(n)} \widetilde{\Gamma}_{(-)}^{M_{1} \cdots M_{n}}$, 
with $\widetilde{\Gamma}_{(-)}^{M}=Q_{-N}^{M} \Gamma^{N}$. To find the transformation of the component field strengths $F^{(n)}$, as well as those of the associated R-R potentials $C^{(n)}$ and $C^{\prime(n)}$, we need the explicit form of the spinor representation $\Omega$ of the Lorentz twist $\Lambda$.

\section{Transformations of R-R Fields}

The T-duality group $O(d, d)$ has elements with determinant +1 and -1 . A representative of the latter class is a single T-duality with respect to a coordinate, say $X^{9}$. This is a $\mathbf{Z}_{2}$ transformation which corresponds to the choice $S=1, R_{N}^{M}=$ $\delta_{N}^{M}-2 \delta_{9}^{M} \delta_{N}^{9}$. It interchanges type-IIA and typeIIB theories 15]. The elements with determinant +1 , of course, form the group $S O(d, d)$ which acts within type-IIA or type-IIB theory. We consider these two cases separately.

\subsection{The Single T-duality Case}

For a single T-duality, say, along $X^{9}$, the spinor representation $\Omega$ is given by [1]

$\Omega=a \sqrt{G_{99}^{-1}} \Gamma_{11} \Gamma_{9}$.

Here, $a$ is an arbitrary sign that we choose to be +1 in going from IIA to IIB and -1 vice versa, so that the transformation squares to +1 . Using this in (21), one obtains

$\widetilde{F}_{9 i_{2} \cdots i_{n}}^{(n)}=-F_{i_{2} \cdots i_{n}}^{(n-1)}+(n-1) G_{99}^{-1} G_{9\left[i_{2}\right.} F_{\left.9 i_{3} \cdots i_{n}\right]}^{(n-1)}$,

$\widetilde{F}_{i_{1} i_{2} \cdots i_{n}}^{(n)}=-F_{9 i_{1} \cdots i_{n}}^{(n+1)}-n B_{9\left[i_{1}\right.} \widetilde{F}_{\left.9 i_{2} \cdots i_{n}\right]}^{(n)}$.

Relation (1) between the $F^{(n)}$ and $C^{(n)}$ then gives

$\widetilde{C}_{9 i_{2} \cdots i_{n}}^{(n)}=C_{i_{2} \cdots i_{n}}^{(n-1)}-(n-1) G_{99}^{-1} G_{9\left[i_{2}\right.} C_{\left.9 i_{3} \cdots i_{n}\right]}^{(n-1)}$,

$\widetilde{C}_{i_{1} i_{2} \cdots i_{n}}^{(n)}=C_{9 i_{1} \cdots i_{n}}^{(n+1)}-n B_{9\left[i_{1}\right.} \widetilde{C}_{\left.9 i_{2} \cdots i_{n}\right]}^{(n)}$,

as first obtained in [4]. The transformation of $C^{\prime(n)}$ can be obtained using $\mathbf{C}^{\prime}=\mathbf{C} \wedge \mathrm{e}^{-B}$, as

$\widetilde{C}_{9 i_{2} \cdots i_{n}}^{\prime(n)}=C_{i_{2} \cdots i_{n}}^{(n-1)}, \quad \widetilde{C}_{i_{1} i_{2} \cdots i_{n}}^{(n)}=C_{9 i_{1} \cdots i_{n}}^{(n+1)}$.

Now we make three simple observations that will greatly simplify things in the next subsection:

- Under a single T-duality, $F^{(n)}$ and $C^{(n)}$ transform in the same way, up to a sign. The sign difference disappears when considering even number of single T-dualities.
- The transformation of $C^{\prime(n)}$ is independent of $G_{M N}$ and $B_{M N}$. Hence, it transforms in the same way as $C^{(n)}$ would in the flat-space $G_{M N} \rightarrow \hat{\eta}_{M N}, B_{M N} \rightarrow 0$.

- Being $n$-forms, $F^{(n)}, C^{(n)}$ and $C^{\prime(n)}$ transform in the same way under $G L(d, R)$ transformations of the coordinates $X^{i}$, in particular, under its $S O(d)$ subgroup.

\subsection{The $S O(d, d)$ Case}

The spinor representation of the Lorentz twist induced by an $S O(d, d)$ transformation of the backgrounds is given by [2]

$\Omega=2^{-\frac{d}{2}} \sqrt{\frac{\operatorname{det}\left(\mathcal{Q}_{-}+\mathcal{Q}_{+}\right)}{\operatorname{det} \mathcal{Q}_{-}}} \circledast\left(-\frac{1}{2} \mathcal{A}^{i j} \Gamma_{i j}\right)$.

Here, $\mathcal{Q}_{ \pm}$stand for the $d \times d$ blocks of $Q_{ \pm}$spanned by the index $i$, and $\Subset$ stands for an exponentiallike expansion with the products of all $\Gamma$-matrices antisymmetrized. The $\mathcal{A}^{i j}$ appearing above is given by

$\mathcal{A}^{i j}=\left[\hat{\eta}_{d}\left(\mathbf{1}_{d}-\mathcal{S}^{-1} \mathcal{R}\right)^{-1}\left(\mathbf{1}_{d}+\mathcal{S}^{-1} \mathcal{R}\right)+\mathcal{B}\right]_{i j}^{-1}$.

Substituting (27) in (21) one obtains

$$
\begin{array}{r}
\widetilde{F}_{M_{1} \cdots M_{n}}=2^{-\frac{d}{2}} \sqrt{\operatorname{det}\left(\mathcal{Q}_{-}+\mathcal{Q}_{+}\right)} \sum_{p=0}^{[d / 2]} \sum_{r=0}^{2 p} \\
\times\left[\frac{(-1)^{p}}{p ! 2^{p}} \frac{(2 p) !}{(2 p-r) !}{ }^{n} C_{r} \mathcal{A}^{\left[i_{1} i_{2}\right.} \cdots \mathcal{A}^{\left.i_{2 p-1} i_{2 p}\right]}\right. \\
\left.G_{i_{1} N_{1}} \cdots G_{i_{r} N_{r}} F_{i_{2 p} \cdots i_{r+1}} N_{r+1} \cdots N_{n}\right] \\
\left.\times\left(Q_{-}^{-1}\right)^{N_{1}} \cdots\left(Q_{-}^{-1}\right)^{N_{n}} M_{n}\right]
\end{array}
$$

where, ${ }^{n} C_{r}$ are the binomial expansion coefficients.

\subsection{Transformation of Potentials $C^{(n)}$}

Unlike the case of a single T-duality, the transformation of $C^{(n)}$ and $C^{\prime(n)}$ cannot be easily worked out by using equation (1). However, these can be obtained by a simple construction combined with the three observations made in subsection 3.1.

Any non-trivial $S O(d, d)$ transformation can, in principle, be constructed as a combination of single T-duality transformations and appropriately chosen coordinate rotations. To see this, let 
$\hat{r}$ denote a unit vector in $d$ dimensions. Any $O(d)$ rotation, say $\mathcal{R}$, can be decomposed as a product of reflections $T_{r_{k}}$ about planes perpendicular to properly chosen axes $\hat{r}_{k}$, i.e., $\mathcal{R}=T_{r_{n}} \cdots T_{r_{1}}$. Equivalently, it can be written as a product of reflections $T_{i}$ about planes perpendicular to the coordinate axes $\hat{x}^{i}$, and properly chosen rotations $\mathcal{O}_{k}$ that rotate the coordinate axes $\hat{x}^{i}$ into the reflection axes $\hat{r}_{k}$. Also setting $\mathcal{S}$ to a product of the rotations $\mathcal{O}_{k}$, we get

$\mathcal{R}=T_{i_{n}} \mathcal{O}_{k_{n}} \cdots T_{i_{1}} \mathcal{O}_{k_{1}}, \quad \mathcal{S}=\mathcal{O}_{k_{n}} \cdots \mathcal{O}_{k_{1}}$.

Then the non-trivial $O(d, d)$ transformation implemented by $\mathcal{R}$ and $\mathcal{S}$ corresponds to a sequence of single T-duality transformations with $\mathcal{R}_{i}=T_{i}$, $\mathcal{S}_{i}=1$, intertwined with coordinate rotations $\mathcal{R}_{k}=\mathcal{S}_{k}=\mathcal{O}_{k}$.

This construction implies that the non-trivial $S O(d, d)$ transformations of $F^{(n)}$ in (29) can, at least in principle, be constructed by applying a succession of single T-dualities (24) and coordinate rotations, using the decomposition (30).

As emphasized in subsection $3.1, F^{(n)}$ and $C^{(n)}$ transform in the same way under a single $\mathrm{T}$ duality (the sign difference is immaterial for even number of T-dualities). They also transform in the same way under coordinate rotations. Therefore, if we construct the action of an $S O(d, d)$ element on $C^{(n)}$, by using its decomposition in terms of single T-dualities and rotations, then we will end up with the same equation as for $F^{(n)}$, i.e., (29), but now with $F^{(n)}$ replaced by $C^{(n)}$. In terms a bispinor $\varnothing$, defined analogously to (5), the transformation is

$\widetilde{\varnothing}=\sqrt{Q_{-}} \Omega \varnothing$.

Needless to say, this formula is also valid for nontrivial $O(d, d)$ transformations.

\subsection{Transformation of Potentials $C^{\prime(n)}$}

As observed in subsection 3.1 , a single Tduality acts on $C^{\prime(n)}$ in the same way as it would act on $C^{(n)}$ in a flat space with $B_{M N} \rightarrow 0$ and $G_{M N} \rightarrow \hat{\eta}_{M N}$. Furthermore, both potentials transform in the same way under coordinate rotations. The decomposition (30) then implies that non-trivial $O(d, d)$ transformations of $C^{\prime(n)}$ are given by the same formula as that for $C^{(n)}$ with $B_{M N}=0$ and $G_{M N}$ set to $\hat{\eta}_{M N}$.

This result can also be written in a compact form similar to equation (31). For this, let us define a set of $S O(1,9)$ Gamma matrices $\hat{\Gamma}^{M}$ by

$\left\{\hat{\Gamma}^{M}, \hat{\Gamma}^{N}\right\}=\hat{\eta}^{M N}$.

The hat indicates that $\hat{\eta}$ is an auxiliary flat metric while the actual space-time metric is still $G_{M N}$. Now we construct the spinor representation of the Lorentz twist $\hat{\Omega}$ in this auxiliary flat space. This is obtained from the expression (27) for $\Omega$ by replacing $\Gamma^{i}$ by $\hat{\Gamma}^{i}$ and setting $B_{i j}=0$. Furthermore, we can combine the $C^{\prime(n)}$ into an $S O(1,9)$ bispinor $\hat{C}^{\prime}$,

$\hat{\varnothing}^{\prime}=\sum_{m} \frac{(-1)^{m}}{m !} C_{M_{1} \cdots M_{m}}^{\prime(m)} \hat{\Gamma}^{M_{1} \cdots M_{m}}$.

Then the non-trivial $S O(d, d)$ transformations of $C^{\prime(n)}$ can be written as

$\tilde{\hat{\varphi}}=\hat{\Omega} \hat{\varnothing}$.

\section{An Application: D-brane Couplings by Clifford Multiplication}

The Abelian theory on a single D-brane couples to the background R-R potentials by exterior multiplication $\mathcal{C}^{\prime} \wedge e^{\mathcal{F}}$, where $\mathcal{C}^{\prime}$ is the pull-back of $\mathbf{C}^{\prime}$ to the worldvolume and $\mathcal{F}$ is the Abelian gauge field strength. In the non-Abelian case the interaction also involves the scalar products of the non-Abelian scalars $\Phi^{i}$ and the R-R potentials. Furthermore, the factors of $\partial X^{i} / \partial \xi^{\alpha}$ appearing in the pull-back of $C^{\prime(n)}$ in the static gauge are replaced by the gauge covariant derivatives $D_{\alpha} \Phi^{i}$ 16,17. These new interactions are written in the static gauge and moreover the replacement of the pull-back by a non-Abelian covariant derivative obscures the geometric description of the D-brane as an embedded surface.

The construction in the last section allows us to go beyond the static gauge and write a covariant and geometric expression that includes the new couplings [3]. This is achieved by replacing the exterior product by a Clifford multiplication defined with respect to the flat metric $\hat{\eta}$ as in (32). To see this, note that the degree of a D-brane 
volume form increases/decreases under a single T-duality transverse/parallel to the brane. Thus, they behave very much like the $C^{\prime(n)}$ in (26) and it makes sense to construct a volume bispinor $\hat{X}$ similar to (33),

$$
\begin{aligned}
\hat{X}= & \sum_{p} \hat{X}^{(p+1)}=\sum_{p} \frac{(-1)^{p+1}}{(p+1) !} T_{(p)} \\
& \times \mathrm{d} X^{L_{1}} \wedge \cdots \wedge \mathrm{d} X^{L_{p+1}} \hat{\Gamma}_{L_{1} \cdots L_{p+1}} .
\end{aligned}
$$

Moreover, the worldvolume gauge fields $\mathcal{A}_{\alpha}(\xi)$ and transverse scalars $\Phi^{I}(\xi)$ can be combined into a 1-form $A_{M}(X(\xi))$ such that

$\mathcal{A}_{\alpha}=A_{M} \partial X^{M} / \partial \xi^{\alpha}, \quad \Phi^{I}=A_{M} \hat{\eta}^{M N} a_{N}^{I}$.

Here, $\partial X^{M} / \partial \xi^{\alpha}$ and $a_{I}^{M}$ span the tangent and normal bundles to the worldvolume ( $I$ is an orthonormal frame index). Let $F$ denote the field strength of $A$ and $\mathbf{Y}=e^{F}$. We can also define a bispinor for the generalized D-brane charges,

$\hat{X}=\sum_{n} \frac{(-1)^{n}}{n !} Y_{N_{1} \cdots N_{n}}^{(n)} \hat{\Gamma}^{N_{1} \cdots N_{n}}$.

It is now easy to write a covariant expression for the Dp-brane couplings to all R-R potentials in terms of a Clifford product,

$I_{W Z}^{(p+1)}=-\operatorname{Str} \int_{\mathcal{W}^{(p+1)}} \operatorname{Tr}\left(\hat{\Gamma}_{11} \overline{\hat{X}} \hat{\Gamma}_{11} \hat{\ell}^{\prime} \hat{Y}\right)$,

where Str is the symmetrized gauge trace and $\mathrm{Tr}$ is a trace over the spinor index. The integral over $\mathcal{W}^{(p+1)}$ restricts the expression to the Dp-brane worldvolume.

The $\Gamma$-matrix multiplication and tracing can be carried out and leads to the component form for the generalized WZ action, including the new interactions. In the static gauge, $\left(X^{\mu}=\xi^{\mu}, X^{i}=\right.$ $X^{i}(\xi)$ it reduces to the known form in 16, 17. The generalized pull-backs discussed above appear as part of the restriction of $F_{M N}$ to the Dbrane which, using (36), gives

$$
\begin{aligned}
D_{\alpha} \Phi^{I}= & \frac{\partial X^{M}}{\partial \xi^{\alpha}}\left(F_{M N}\right) \hat{\eta}^{N L} a_{L}^{I} \\
& =\partial_{\alpha} \Phi^{I}+\Theta_{\alpha J}^{I} \Phi^{J}+\left[\mathcal{A}_{\alpha}, \Phi^{I}\right] .
\end{aligned}
$$

The appearance of the normal bundle connection $\Theta_{\alpha J}^{I}=a_{M}^{I} \partial_{\alpha} a_{J}^{M}$ here is a reflection of the covariance of the formalism. However, it does not yet contain the contribution from curved backgrounds for which it may be necessary to consider the gravitational couplings of the brane.

It is a pleasure to thank the organizers of the International Conference on "Supersymmetry and Quantum Field Theory" (Kharkov, July 25$29,2000)$ for the invitation and hospitality.

\section{REFERENCES}

1. S. F. Hassan, Nucl. Phys. B 568 (2000) 145 hep-th/9907152.

2. S. F. Hassan, Nucl. Phys. B 583 (2000) 431 hep-th/9912236.

3. S. F. Hassan and R. Minasian, "D-brane couplings, RR fields and Clifford multiplication," hep-th/0008149.

4. E. Bergshoeff, C. Hull and T. Ortin, Nucl. Phys. B 451 (1995) 547 hep-th/9504081.

5. M. Cvetic, H. Lu, C. N. Pope and K. S. Stelle, Nucl. Phys. B 573 (2000) 149 hep-th/9907202.

6. K. Kamimura and J. Simon, Nucl. Phys. B 585 (2000) 219 hep-th/0003211.

7. B. Kulik and R. Roiban, "T-duality of the Green-Schwarz superstring," hepth/0012010.

8. C. M. Hull and P. K. Townsend, Nucl. Phys. B 438 (1995) 109 hep-th/9410167.

9. E. Witten, Nucl. Phys. B 443 (1995) 85 hepth/9503124.

10. M. Fukuma, T. Oota and H. Tanaka, Prog. Theor. Phys. 103 (2000) 425 hepth/9907132.

11. Y. Gong, "Ramond-Ramond field transformation," hep-th/0002133.

12. J. H. Schwarz, Nucl. Phys. B 226 (1983) 269.

13. L. J. Romans, Phys. Lett. B 169 (1986) 374.

14. S. F. Hassan, Nucl. Phys. B 454 (1995) 86 hep-th/9408060.

15. M. Dine, P. Huet and N. Seiberg, Nucl. Phys. B 322 (1989) 301.

16. W. I. Taylor and M. Van Raamsdonk, Nucl. Phys. B 573 (2000) 703 hep-th/9910052.

17. R. C. Myers, JHEP9912 (1999) 022 hepth/9910053. 